\title{
MR Imaging-Detected Carotid Plaque Hemorrhage Is Stable for 2 Years and a Marker for Stenosis Progression
}

\author{
R.J. Simpson, S. Akwei, A.A. Hosseini, S.T. MacSweeney, D.P. Auer, and N. Altaf
}

\begin{abstract}
BACKGROUND AND PURPOSE: MR imaging-detected carotid plaque hemorrhage is associated with an increased risk of recurrent ischemic cerebrovascular events and could be an indicator of disease progression; however, there are limited data regarding the dynamics of the MR imaging-detected carotid plaque hemorrhage signal. We assessed the temporal change of this signal and its impact on carotid disease progression.
\end{abstract}

MATERIALS AND METHODS: Thirty-seven symptomatic patients with 54 carotid stenoses of $>30 \%$ on sonography underwent serial MR imaging during 24 months. A signal-intensity ratio of $>1.5$ between the carotid plaque and adjacent muscle was defined as plaque hemorrhage, and a change in signal-intensity ratio of $>0.31$ between time points was considered significant. Sixteen patients underwent $\geq 2$ carotid sonography scans to determine the peak systolic velocities and degree of stenosis with time.

RESULTS: Of the 54 carotids, 28 had the presence of hyperintense signal on an MR imaging sequence $(\mathrm{PH}+)$ and 26 had the absence of hyperintense signal on an MR imaging sequence $(\mathrm{PH}-$ ) at baseline. The signal-intensity ratio was stable in 33/54 carotid plaques, but $39 \%$ showed a change. Plaque hemorrhage classification did not change in $87 \%$ of carotid plaques, but 4 became $\mathrm{PH}+$, and $3, \mathrm{PH}-$. As a group, $\mathrm{PH}+$ carotids did not change significantly in signal-intensity ratio $(P=.585)$, whereas $\mathrm{PH}-$ showed an increased signal-intensity ratio at 24.5 months $(P=.02)$. In $\mathrm{PH}+$ plaques, peak systolic velocities significantly increased by $22 \pm 39.8 \mathrm{~cm} / \mathrm{s}$ from baseline to last follow-up sonography $(Z=2.427, P=.013)$.

CONCLUSIONS: During 2 years, MR imaging-detected carotid plaque hemorrhage status remained stable in most ( $87 \%)$ cases with 4 (7\%) incident plaque hemorrhages. $\mathrm{PH}+$ plaques were associated with increased flow velocity during the follow-up period.

ABBREVIATIONS: MR imaging-PH = MR imaging-detected plaque hemorrhage; $\mathrm{PH}=$ plaque hemorrhage; $\mathrm{PH}+=$ presence of hyperintense signal on an $\mathrm{MR}$ imaging sequence; $\mathrm{PH}-=$ absence of hyperintense signal on an MR imaging sequence; PSV = peak systolic velocity; SIR = signal-intensity ratio

C urrently, the degree of ICA stenosis is the principal criterion on the basis of which the decision for carotid intervention is made. This is based on strong evidence from randomized con-

Received October 14, 2014; accepted after revision November 12.

From the Radiological Sciences Group (R.J.S., S.A., A.A.H., D.P.A., N.A.), Division of Clinical Neurosciences, University of Nottingham, Nottingham, United Kingdom; and Department of Vascular and Endovascular Surgery (R.J.S., S.T.M., N.A.), Nottingham University Hospitals National Health Service Trust, Nottingham, United Kingdom.

R.J.S. and S.A. contributed equally to this work.

This work was supported by the National Institute for Health Research under its Research for Patient Benefit Programme (grant reference number PB-PG-010711438). Financial support was also provided by the Stroke Association Junior Research Training Fellowship, London, United Kingdom; the Nottingham Vascular Surgery Research Fund, Nottingham, United Kingdom; and The Health Foundation, London, United Kingdom.

The views expressed are those of the authors and not necessarily those of the National Health Service, the National Institute for Health Research, or the Department of Health.

Paper previously presented at: UK Stroke Forum 2011, November 29-December 1, 2011; Glasgow, United Kingdom. trolled trials that carotid endarterectomy reduces stroke risk in patients with severe carotid artery stenosis. ${ }^{1,2}$ However, those studies also showed that a significant proportion of patients with symptomatic carotid disease will not have a recurrence. Subsequently, much research is focused on the identification of highrisk subgroups, ${ }^{3}$ especially for those with moderate or asymptomatic carotid stenosis.

Plaque hemorrhage $(\mathrm{PH})$ is implicated in carotid plaque vulnerability ${ }^{4}$ and is detectable by MR imaging. ${ }^{5,6}$ A recent longitudinal follow-up study and meta-analysis ${ }^{7}$ demonstrated that MR imaging-detected plaque hemorrhage (MR imaging- $\mathrm{PH}$ ) strongly predicts recurrent ischemic events. The MR imaging-PH

Please address correspondence to Richard J. Simpson, Radiological Sciences, Division of Clinical Neurosciences, University of Nottingham, B Floor, West Block, Queens Medical Centre, Nottingham, NG7 2UH, UK; e-mail: msxrs@nottingham.ac.uk

- Indicates open access to non-subscribers at www.ajnr.org

http://dx.doi.org/10.3174/ajnr.A4267 
Table 1: Velocity grading criteria based on the NASCET angiographic method ${ }^{13,1}$

\begin{tabular}{cccc}
\hline Stenosis & $\begin{array}{c}\text { ICA PSV } \\
\text { (cm/s) }\end{array}$ & $\begin{array}{c}\text { ICA EDV } \\
\text { (cm/s) }\end{array}$ & $\begin{array}{c}\text { CCA/ICA } \\
\text { PSV Ratio }\end{array}$ \\
\hline $0-29$ & $<100$ & $<40$ & $<3.2$ \\
$30-49$ & $110-130$ & $<40$ & $<3.2$ \\
$50-59$ & $>130$ & $<40$ & $<3.2$ \\
$60-69$ & $>130$ & $40-110$ & 3.2 to $<4.0$ \\
$70-79$ & $>210$ & $120-140$ & $\leq 4.0$ \\
$80-95$ & $>210$ & $>140$ & $\leq 4.0$ \\
$96-99$ & String flow & String flow & String flow \\
100 & Occluded & Occluded & Occluded \\
\hline
\end{tabular}

Note:-CCA indicates common carotid artery; EDV, end diastolic velocity.

signal seems to be stable for 12 months, ${ }^{8}$ but stability of MR imaging- $\mathrm{PH}$ features beyond 12 months remains unclear. Knowledge of longer term stability of MR imaging-PH would be helpful if it is to be used to assist decision-making in interventions and to determine the need for follow-up imaging.

Plaque volume progression on sonography recently has been shown to predict cerebrovascular events ${ }^{9}$; however, this measure was not used in the current study. Carotid stenosis progression has been suggested to be a better predictor of subsequent TIA/ stroke than a single measurement. ${ }^{10}$ Although general carotid sonography surveillance may not be cost-effective, ${ }^{11}$ this situation may well be different for a subgroup of patients with a higher risk for stenosis progression. It is conceivable that MR imaging- $\mathrm{PH}$ is also an indicator of disease progression ${ }^{12}$ and, therefore, may be useful in this regard.

The aim of this study was to determine MR imaging signal changes in the carotid artery plaque during 2 years and whether the presence of MR imaging- $\mathrm{PH}$ at baseline is associated with stenosis progression.

\section{MATERIALS AND METHODS}

Eligible patients presenting with a recent history of nondisabling stroke, hemispheric transient ischemic attack, or amaurosis fugax and ipsilateral carotid stenosis of $>30 \%$ measured by sonography were recruited into this study from a TIA clinic. Peak systolic velocity (PSV) and end diastolic velocity measurements were recorded from the common, internal, and external carotids bilaterally. Established grading criteria were used to assess the percentage stenosis (Table 1), which was taken from the Carotid and Vertebral Artery Transluminal Angioplasty Study ${ }^{13,14}$ and was based on the NASCET angiographic method. ${ }^{2}$ A planned carotid intervention and atrial fibrillation were exclusion criteria. In addition, contralateral carotid plaques were included if a stenosis of $>30 \%$ was observed. Patients were scheduled to undergo serial MR imaging and sonography. MRI and clinical assessment to determine evidence of recurrence were performed at baseline and at 4,12 , and 24 months.

All patients continued to receive routine clinical care independent of their MR imaging findings. The local research ethics committee approved the study, and all patients gave written informed consent at the point of recruitment. The data regarding recurrence have been published previously. ${ }^{15,16}$

\section{MR Imaging Protocol and Image Analysis}

MR carotid imaging was undertaken on a 1.5T Magnetom Vision scanner (Siemens, Erlangen, Germany) by using a receive-only quadrature neck array coil. The MR imaging, previously described, ${ }^{6}$ was a coronal T1-weighted magnetization-prepared 3D gradient-echo sequence incorporating a selective number of partitions, a water-excitation pulse, and a TI chosen to null the signal from blood and fat (TR, $10.3 \mathrm{~ms}$; TE, $4.0 \mathrm{~ms}$; flip angle, $15^{\circ}$; TI, 20 ms; FOV, $350 \times 300 \mathrm{~mm}$; matrix, $256 \times 140$; 140 partitions; volume thickness, $120-150 \mathrm{~mm}$ ). This sequence detects subacute-to-chronic hemorrhage because methemoglobin has a hyperintense signal.

MR imaging analysis was performed on standard reformatted axial images by using Java imaging software (www.xinapse. com), by 3 reviewers with 1 - to 3 -years' carotid image-analysis experience, blinded to clinical data and images from other time points. As previously described, ${ }^{15-17}$ the highest intensity in the carotid artery wall within $1 \mathrm{~cm}$ from the bifurcation was compared with all of the immediately adjacent sternocleidomastoid muscle (signal-intensity $[\mathrm{SI}]$ ratio $[\mathrm{SIR}]=S I_{\text {plaque }} / S I_{\text {muscle }}$ ). The presence of MR imaging- $\mathrm{PH}$ was diagnosed if the normalized SIR between the 2 was at least $1.5(\mathrm{PH}+)$. Inter- and intraobserver agreements were assessed by analyzing all the images 5 months after the initial analysis. The minimum detectable change applied to the SIR is considered the minimal amount of change in SIR to exceed an estimated error due to variation in measurement. Thus a change in SIR is only considered a real change if it is larger than the threshold set at \pm 0.31 , defined as twice the pooled SD of intraobserver test-retest data. A change in $\mathrm{PH}$ status from $\mathrm{PH}-$ (normalized SIR of $<1.5$ ) to $\mathrm{PH}+$ was noted only when the SIR rose above the 1.5 threshold and vice versa.

\section{Sonography}

All carotid sonography (baseline and follow-up) was performed by 1 of 2 accredited Clinical Vascular Scientists by using a Logiq 9 sonography machine (GE Healthcare, Milwaukee, Wisconsin). The ultrasound grading criteria in the follow-up stage were identical to that of the recruitment stage, as detailed above. Change in peak systolic velocity in the internal carotid artery from baseline to the last follow-up sonography scan was used as the primary marker of progressive carotid disease. ${ }^{18}$ Categoric progression of stenosis, where there was an increase by at least 1 category, was also reported.

\section{Statistical Analysis}

SIR inter-/intraobserver agreement was analyzed by using the Cronbach $\alpha$. The SIR at different time points was analyzed with repeated-measures analysis of variance, in which if the Mauchly test of sphericity was not significant, then the Geenhouse-Geisser adjustment was reported. The $\chi^{2}$ test was used to compare the prevalence of $\mathrm{PH}+$ at baseline between symptomatic and asymptomatic carotids. The Fisher exact test was used to determine the association between disease progression and both the presence of $\mathrm{PH}$ and the recurrence of symptoms. The Wilcoxon signed rank test was used to test the effect of PH on PSV of the ICA. All analyses were performed with SPSS Statistics for Windows, Version 19.0 (IBM, Armonk, New York). $P<.05$ was considered statistically significant. 


\section{RESULTS}

Sixty-four participants were recruited during a 2-year period, but 27 were excluded because they did not undergo subsequent MR imaging (18 with claustrophobia, 3 with bilateral stenosis $<30 \%$, 6 "other"). Therefore, 37 patients were included in this study; their demographics can be seen in Table 2, and carotid stenosis at baseline, in Table 3.

At baseline, 28/54 (52\%) carotid arteries were $\mathrm{PH}+$ (mean SIR, $2.02 \pm 0.39)$. The mean SIR of those without MR imaging- $\mathrm{PH}$ was $1.31 \pm 0.13$. $\mathrm{PH}$ was detected in $22 / 37$ (59\%)

Table 2: Patient characteristics

\begin{tabular}{lc}
\hline \multicolumn{1}{c}{ Characteristic } & \\
\hline Age (yr) (mean) (SD) & $70.5(9.24)$ \\
Male & $87 \%$ \\
Smoking history & \\
$\quad$ Never & $8(22 \%)$ \\
Previous smoker & $16(43 \%)$ \\
$\quad$ Current smoker & $13(35 \%)$ \\
Ischemic heart disease & $6(16 \%)$ \\
Diabetes & $8(22 \%)$ \\
High cholesterol & $20(54 \%)$ \\
Hypertensive & $29(78 \%)$ \\
Statin use & \\
$\quad$ None & $12(32 \%)$ \\
Commenced after TIA/stroke & $25(68 \%)$ \\
\hline
\end{tabular}

Table 3: Degree of carotid stenosis at baseline ${ }^{a}$

\begin{tabular}{ccc}
\hline Degree of stenosis & Ipsilateral ICA & Contralateral ICA \\
\hline $0 \%-30 \%$ & - & $20(54.1)$ \\
$30 \%-49 \%$ & $10(27.0)$ & $4(10.8)$ \\
$50 \%-59 \%$ & $4(10.8)$ & $1(2.7)$ \\
$60 \%-69 \%$ & $19(51.4)$ & $3(8.1)$ \\
$70 \%-79 \%$ & $1(2.7)$ & - \\
$80 \%-95 \%$ & $1(2.7)$ & $4(10.8)$ \\
$95 \%-99 \%$ & $2(5.4)$ & - \\
$100 \%$ & - & $5(13.5)$ \\
\hline
\end{tabular}

Note:- - indicates none.

a Values are counts (percentages). "Ipsilateral" and "Contralateral" are used to describe the carotid artery in relation to presenting symptoms.

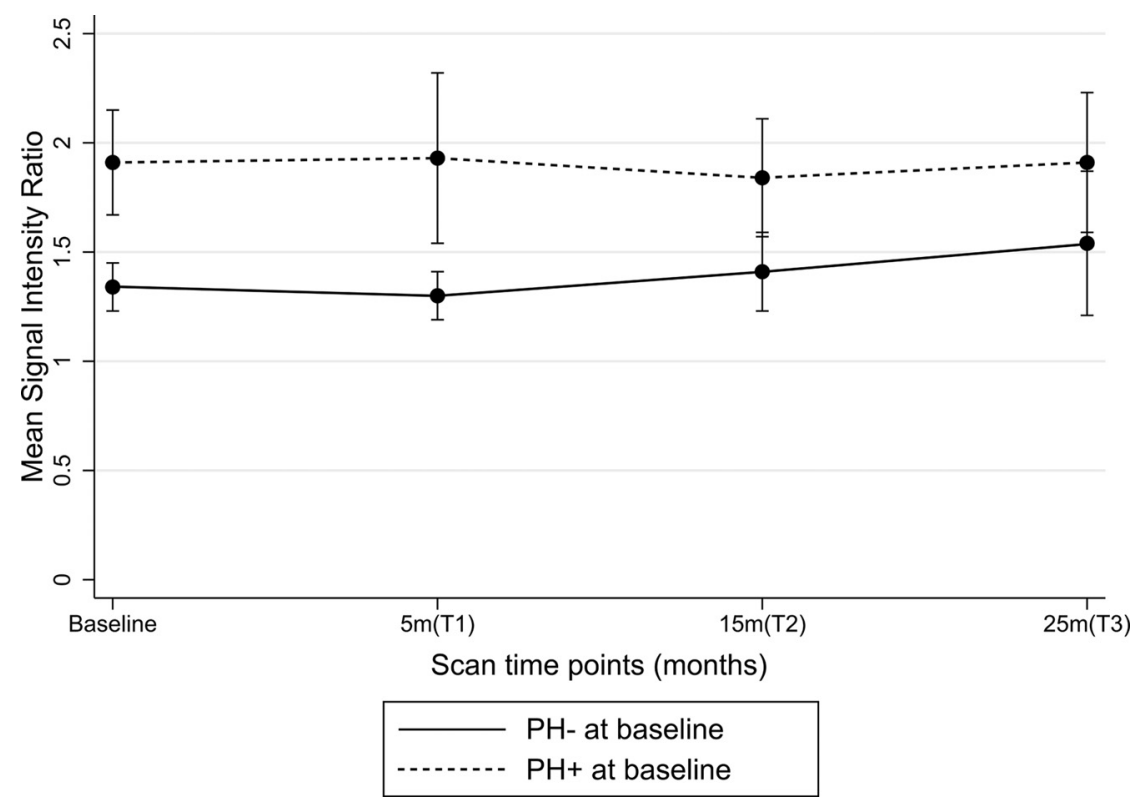

FIGURE. Trend of SIR during 24.5 months based on baseline PH status. symptomatic carotid arteries, compared with 6/17 (35\%) in the asymptomatic group; however, this difference was not significant $\left(\chi^{2}=2.7, P=.1\right)$.

Thirty-seven patients with 54 carotid plaques with $>30 \%$ stenosis (37 symptomatic and 17 asymptomatic) underwent serial MR imaging. The mean times for follow-up scans from baseline were T1 (4.5 \pm 3.1 months), T2 (14.5 \pm 3.1 months), and T3 $(24.6 \pm 2.6$ months $)$. Suitable images were available for all carotid arteries at T0 (baseline), but only 46, 47, and 34 at T1, T2, and T3, respectively, due to loss to follow-up and uninterpretable images. Thus, $84 \%(181 / 216)$ of the potential images were included in the analysis.

\section{Temporal Stability of MR Imaging PH}

The carotid muscle SIR was stable with time in 33 of the 54 carotid arteries (61\%). Thus, 21 carotid plaques $(16 \mathrm{PH}+$ and $5 \mathrm{PH}-$ ) underwent a detectable change in SIR of $>0.31$ absolute difference at some point during follow-up. Most of the detectable changes (14/21) resulted in an increase in SIR; of these, $13 \mathrm{PH}+$ plaques remained $\mathrm{PH}+(\mathrm{SIR}>1.5)$, while $1 \mathrm{PH}-$ plaque remained $\mathrm{PH}-(\mathrm{SIR}<1.5)$, despite the change. This means that $\mathrm{PH}$ status remained the same in $47 / 54$ plaques $(87 \%)$. The 7 plaques demonstrating categoric change included 4 of 26 (7\%) previously $\mathrm{PH}-$ plaques that became $\mathrm{PH}+$, while 3 of $28(5 \%)$ with $\mathrm{PH}+$ reverted to $\mathrm{PH}-$.

At the group level, SIR change did not differ with symptom status at baseline. SIR significantly increased during a mean of $24.5 \pm 2.56$ months $(P=.02$, repeated-measures ANOVA $)$ in $\mathrm{PH}-$ plaques (Figure). However, SIR did not significantly increase during the follow-up period in $\mathrm{PH}+$ plaques $(P=.585)$. Quantitative assessment of SIR showed excellent reliability on both interobserver (Cronbach $\alpha=0.99$ ) and intraobserver (Cronbach $\alpha=0.99$ ) assessment.

\section{Recurrent Events}

As previously reported, ${ }^{15} 11$ patients (30\%) had recurrent symptoms (stroke, 7; amaurosis fugax, 2; TIA, 2) during follow-up or within 6 months of their final scan. Most, 9/11 (82\%), recurrent symptoms occurred in the previously symptomatic carotid artery. Five events occurred in carotid plaques that had undergone detectable positive change in SIR. No events occurred in plaques with new $\mathrm{PH}+$. There was, however, a significant association between recurrent events and baseline plaque hemorrhage status. Nine $(82 \%)$ events occurred in the 28 carotid arteries that were $\mathrm{PH}+$ at baseline compared with 2 events in the $26 \mathrm{PH}-$ carotid arteries at baseline, $\left(\chi^{2}, P=.026\right)$.

\section{Carotid Stenosis Progression}

A subgroup of 16 patients with $\geq 30 \%$ ipsilateral stenosis and variable contralateral disease were followed up with carotid sonography during a mean of 
40.2 months (range, 20-91 months; 46 sonograms). However, due to limited access to the sonography machine, it was not possible to scan all patients. The mean scan intervals from baseline were the following: T1 $(26.1 \pm 13.01$ months $), \mathrm{T} 2(34.1 \pm 10.19$ months), and T3 (55.3 \pm 26.85 months). At the group level, there was a significant progression of ICA-PSV in $\mathrm{PH}+$ carotid arteries, with a mean increase of $22 \pm 39.8 \mathrm{~cm} / \mathrm{s}$ from baseline $(147 \mathrm{~cm} / \mathrm{s})$ to last follow-up $(169 \mathrm{~cm} / \mathrm{s})$ sonogram $(Z=2.427, P=.013)$. No such association was seen in plaques without $\mathrm{MR}$ imaging- $\mathrm{PH}$ $(-5.5 \pm 55.4 \mathrm{~cm} / \mathrm{s})$. Symptomatic carotid stenoses were not associated with an increase in ICA PSV.

Stenosis progression according to predefined grades was seen in 9 of the 32 carotid arteries (28\%), giving an annualized progression rate of $8.4 \%$. However, no significant differences were seen in the grade progression between symptomatic (5/16) and asymptomatic (4/16) plaques or between $\mathrm{PH}+(6 / 22)$ and $\mathrm{PH}-$ (3/10) disease.

\section{DISCUSSION}

MR imaging- $\mathrm{PH}$-positive carotid plaques showed no change in SIR during 24 months but demonstrated a significant increase in peak systolic velocities. In contrast, there was no progression of velocities but a mild increase in MR imaging signal intensity in $\mathrm{PH}-$ plaques.

In $28 \mathrm{PH}+$ plaques, there was no change in relative MR imaging signal intensity during 24 months, providing further evidence of the stability of PH. The observed prolonged elevation of signal suggests persistence of methemoglobin, in line with previous reports on MR imaging-PH for up to 18 months. ${ }^{8,19,20}$ We also showed stable plaque signal in asymptomatic plaques, which corroborates a small study in 14 asymptomatic patients with $\mathrm{PH}$ signs for 54 months. ${ }^{21}$ However, selection bias in that study should be noted because only those plaques with $\mathrm{MR}$ imaging- $\mathrm{PH}$ on the most recent scan were included.

Quantitative assessment of plaque intensities revealed a small but significant increase in previously plaque hemorrhage-negative plaques. In contrast to our findings, Wang et $\mathrm{al}^{22}$ recently reported a reduction in SIR during 19 months in asymptomatic plaques. These differences may be explained by differences in the selection of asymptomatic patients with carotid disease between studies. We defined an asymptomatic plaque as contralateral to the symptomatic artery and symptom-free for $>6$ months, whereas Wang et $\mathrm{al}^{22}$ selected patients who had never had any symptoms of cerebrovascular disease. Evidence suggests that differences exist between these 2 groups, in that truly asymptomatic plaques appear to be more stable. ${ }^{23}$ Conversely, the overall SIR increase in $\mathrm{PH}$ plaques is corroborated by the individual classification showing that $15 \%$ of $\mathrm{PH}$ plaques converted during the follow-up of 2 years. The most likely explanation is the incident plaque hemorrhage in a higher risk patient group than in a completely asymptomatic patient cohort.

Although the MR imaging signal intensity due to intracranial hemorrhage is subject to rapid change, ${ }^{24}$ carotid MR imaging- $\mathrm{PH}$ and thus methemoglobin have been shown to be more persistent. ${ }^{19}$ The temporal stability of MR imaging-PH may be due to the inherent stability of methemoglobin or because its breakdown is balanced by continued production of methemoglobin from continued hemorrhage from a fragile plaque vasa vasorum. ${ }^{25}$ Our results indicating temporal stability of the MR imaging- $\mathrm{PH}$ signal, together with previous work that reported MR imaging- $\mathrm{PH}$, strongly predict recurrent stroke/ $\mathrm{TIA}^{7}$ and raise the possibility that all plaques found to be $\mathrm{PH}+$ reflect a cumulative high risk regardless of how recent the $\mathrm{PH}$ may have been. This is particularly pertinent for delayed presentations of TIA and stroke. The evidence of incident $\mathrm{PH}$ certainly suggests that carotid disease merits surveillance; however, the natural history of newly formed $\mathrm{PH}$ in carotid plaques remains uncertain.

We found an increase in PSV in $\mathrm{PH}+$ but not in $\mathrm{PH}-$ carotid arteries, which did, however, not translate into a preferential progression in the categoric degree of stenosis. Although clinically useful, the categorization of blood flow velocities and ratios (Table 1) has the effect of reducing the statistical power to detect the relationship among variables. ${ }^{26}$ Thus, plaque area/volume, PSV, and other continuous variables may be more sensitive to changes in disease progression than grades of stenosis. Our findings, while preliminary, suggest that $\mathrm{PH}$ may be a maker of stenosis progression. This would be in line with a previous study that showed ${ }^{21}$ that the presence of $\mathrm{MR}$ imaging- $\mathrm{PH}$ increased the rate of plaque progression determined by wall volume. Histologic evidence from coronary artery plaques also supports the notion that the presence of $\mathrm{PH}$ increases atheroma progression. ${ }^{12}$

\section{Study Limitations}

Our study is limited by its sample size; thus, there were a relatively small number of carotid plaques that changed PH status or progressed during the follow-up. This finding was further confounded by the exclusion bias for patients scheduled for carotid intervention, meaning that our cohort either had minor carotid disease or those with more severe disease had declined or were turned down for surgery. The small sample size has resulted in wide SD for the mean changes in internal carotid artery peak systolic velocity. A number of scans were not available for analysis due to loss to follow-up and poor-quality images, despite multiple invitations for the non-attenders. In this study, we used a single T1-weighted imaging sequence to detect $\mathrm{PH}$, to optimize its clinical applicability. This has meant that we did not use multisequence imaging to determine the age of the $\mathrm{PH}$, as used by Chu et $\mathrm{al}^{27}$ and Wang et al. ${ }^{22} \mathrm{We}$ also did not measure the volume of the MR imaging- $\mathrm{PH}$ with time, due to the limited resolution of the images at $1.5 \mathrm{~T}$. Although sonography-derived plaque volume is also a marker of vulnerability, ${ }^{9}$ no measurement of volume was made in our study. In addition, quantification of plaque volume has been demonstrated recently by using serial CT angiography. ${ }^{28}$

\section{CONCLUSIONS}

These results demonstrate the temporal stability of carotid MR imaging- $\mathrm{PH}$, supporting its value as a risk marker beyond the acute presentation. We also provide preliminary evidence that $\mathrm{PH}$ may be a marker for stenosis progression as measured by PSV.

Disclosures: Richard J. Simpson—RELATED: Grant: Stroke Association, ${ }^{*}$ National Institute for Health Research, ${ }^{\star}$ Comments: Junior Research Training Fellowship, National Institute for Health Research-Research for Patient Benefit program. Solomon Akwei-UNRELATED: Travel/Accommodations/Meeting Expenses Unrelated to Activities Listed: Nottingham Vascular Research Fund, Comments: covered expenses 
incurred for attending the Vascular Society Meeting, Brighton, UK, November 2010. Akram A. Hosseini-RELATED: Grant: National Institute for Health Research, ${ }^{*}$ Comments: National Institute for Health Research Clinical Research Fellowship. Dorothee P. Auer-RELATED: Grant: Stroke Association, UK, ${ }^{\star}$ Comments: paid for Richard Simpson's fellowship; Support for Travel to Meetings for the Study or Other Purposes: Stroke Association, UK, ${ }^{\star}$ Comments: travel and consumables for Richard Simpson; UNRELATED: Grants/Grants Pending: National Institute of Health Research-Research for Patient Benefit program, ${ }^{*}$ MS Society, ${ }^{*}$ Parkinson's UK, ${ }^{*}$ Medical Research Council, ${ }^{*}$ Engineering and Physical Sciences Research Council, ${ }^{\star}$ Arthritis Research UK, ${ }^{*}$ Cancer Research UK. ${ }^{*}$ Money paid to the institution.

\section{REFERENCES}

1. European Carotid Surgery Trialists' Collaborative Group. MRC European Carotid Surgery Trial: interim results for symptomatic patients with severe (70-99\%) or with mild (0-29\%) carotid stenosis. Lancet 1991;337:1235-43

2. North American Symptomatic Carotid Endarterectomy Trial Collaborators. Beneficial effect of carotid endarterectomy in symptomatic patients with high-grade carotid stenosis. N Engl J Med 1991; 325:445-53

3. U-King-Im J, Young V, Gillard JH. Carotid-artery imaging in the diagnosis and management of patients at risk of stroke. Lancet Neurol 2009;8:569-80

4. Stary HC, Chandler AB, Dinsmore RE, et al. A definition of advanced types of atherosclerotic lesions and a histological classification of atherosclerosis: a report from the Committee on Vascular Lesions of the Council on Arteriosclerosis, American Heart Association. Circulation 1995;92:1355-74

5. Moody AR, Allder S, Lennox G, et al. Direct magnetic resonance imaging of carotid artery thrombus in acute stroke. Lancet 1999;353:122-23

6. Moody AR, Murphy RE, Morgan PS, et al. Characterization of complicated carotid plaque with magnetic resonance direct thrombus imaging in patients with cerebral ischemia. Circulation 2003;107: 3047-52

7. Hosseini AA, Kandiyil N, Macsweeney ST, et al. Carotid plaque hemorrhage on magnetic resonance imaging strongly predicts recurrent ischemia and stroke. Ann Neurol 2013;73:774-84

8. Kwee RM, van Oostenbrugge RJ, Mess WH, et al. Carotid plaques in transient ischemic attack and stroke patients: one-year follow-up study by magnetic resonance imaging. Invest Radiol 2010;45:803-09

9. van Engelen A, Wannarong T, Parraga G, et al. Three-dimensional carotid ultrasound plaque texture predicts vascular events. Stroke 2014;45:2695-701

10. Bertges DJ, Muluk V, Whittle J, et al. Relevance of carotid stenosis progression as a predictor of ischemic neurological outcomes. Arch Intern Med 2003;163:2285-89

11. Cull DL, Cole T, Miller B, et al. The value of a carotid duplex surveillance program for stroke prevention. Ann Vasc Surg 2011;25:887-94

12. Kolodgie FD, Gold HK, Burke AP, et al. Intraplaque hemorrhage and progression of coronary atheroma. N Engl J Med 2003;349:2316-25

13. McCabe DJ, Pereira AC, Clifton A, et al. Restenosis after carotid angioplasty, stenting, or endarterectomy in the Carotid and Verte- bral Artery Transluminal Angioplasty Study (CAVATAS). Stroke 2005;36:281-86

14. Sidhu PS, Allan PL. Ultrasound assessment of internal carotid artery stenosis. Clin Radiol 1997;52:654-58

15. Altaf N, Daniels L, Morgan PS, et al. Detection of intraplaque hemorrhage by magnetic resonance imaging in symptomatic patients with mild to moderate carotid stenosis predicts recurrent neurological events. J Vasc Surg 2008;47:337-42

16. Kandiyil N, Altaf N, Hosseini AA, et al. Lower prevalence of carotid plaque hemorrhage in women, and its mediator effect on sex differences in recurrent cerebrovascular events. PLoS One 2012;7:e47319

17. Altaf N, MacSweeney ST, Gladman J, et al. Carotid intraplaque hemorrhage predicts recurrent symptoms in patients with high-grade carotid stenosis. Stroke 2007;38:1633-35

18. Schillinger M, Exner M, Mlekusch W, et al. Inflammation and Carotid Artery-Risk for Atherosclerosis Study (ICARAS). Circulation 2005;111:2203-09

19. Takaya N, Yuan C, Chu B, et al. Presence of intraplaque hemorrhage stimulates progression of carotid atherosclerotic plaques: a highresolution magnetic resonance imaging study. Circulation 2005; 111:2768-75

20. Saam T, Yuan C, Chu B, et al. Predictors of carotid atherosclerotic plaque progression as measured by noninvasive magnetic resonance imaging. Atherosclerosis 2007;194:e34-42

21. Sun J, Underhill HR, Hippe DS, et al. Sustained acceleration in carotid atherosclerotic plaque progression with intraplaque hemorrhage: a long-term time course study. JACC Cardiovasc Imaging 2012;5:798-804

22. Wang Q, Wang Y, Cai J, et al. Differences of signal evolution of intraplaque hemorrhage and associated stenosis between symptomatic and asymptomatic atherosclerotic carotid arteries: an in vivo high-resolution magnetic resonance imaging follow-up study. Int $J$ Cardiovasc Imaging 2010;26:323-32

23. van Lammeren GW, den Hartog AG, Pasterkamp G, et al. Asymptomatic carotid artery stenosis: identification of subgroups with different underlying plaque characteristics. Eur J Vasc Endovasc Surg 2012;43:632-36

24. Bradley WG Jr. MR appearance of hemorrhage in the brain. Radiology 1993;189:15-26

25. Virmani R, Kolodgie FD, Burke AP, et al. Atherosclerotic plaque progression and vulnerability to rupture: angiogenesis as a source of intraplaque hemorrhage. Arterioscler Thromb Vasc Biol 2005; 25:2054-61

26. Altman DG, Royston $P$. The cost of dichotomising continuous variables. BMJ 2006;332:1080

27. Chu B, Kampschulte A, Ferguson MS, et al. Hemorrhage in the atherosclerotic carotid plaque: a high-resolution MRI study. Stroke 2004;35:1079-84

28. van Gils MJ, Vukadinovic D, van Dijk AC, et al. Carotid atherosclerotic plaque progression and change in plaque composition over time: a 5-year follow-up study using serial CT angiography. AJNR Am J Neuroradiol 2012;33:1267-73 